\title{
Diritti umani ed etica del lavoro nella prospettiva della responsabilità sociale dell'impresa
}

\author{
Laura Solieri \\ Modena, Mo, Italia \\ laurasolieri@yahoo.it \\ Scuola Internazionale di \\ Dotoratto in Relazioni di Lavoro.
}

Recebido em 16/11/2010

Aprovado em 24/03/2011

\section{Resumo}

In questo articolo, si cerca di delineare nell'ambito dei diritti umani fondamentali in materia di lavoro, la prospettiva rappresentata dalle prassi di Responsabilità sociale dell'impresa, la cui peculiarità è quella di andare ad incidere sia sul piano giuridico che sul piano economico che, inevitabilmente, sul piano sociale. In questo contesto, si dedica un approfondimento al ruolo dei codici di condotta sottolineando

come l'autoregolamentazione possa costituire una soluzione efficace per mettere in comunicazione la sfera economica con i valori sociali nell'era della globalizzazione.

\section{Parole-chiave}

Responsabilità sociale dell'impresa. Diritti umani fondamentali in materia di lavoro. Codici di condotta. Autoregolamentazione. Diritto del lavoro. Cooperazione. Etica. Legge. 


\title{
Human rights and ethics of work in the perspective of corporate social responsibility
}

\author{
Laura Solieri
}

Abstract

This article tries to outline in the area of interest of fundamental labor rights, the perspective represented by the routine procedures of Corporate social responsibility. The peculiarity of CSR is its influence both on the juridical plane and the economical plane and, inevitably, on the social plane as well. In this context, an in-depth examination is dedicated to the role of codes of conduct, the instruments par excellence of Csr's practices, highlighting how self-regulation can constitute an effective solution to connect the economical sphere to social values in the globalization era.

Key words

Corporate Social Responsability. Fundamental buman labor rights. Codes of conduct. Selfregulation. Labor law. Cooperation. Ethics. Law. 


\section{Indice}

1 Organizzazione Internazionale del Lavoro e Nazioni Unite contro la discriminazione nel lavoro

2 La Responsabilità sociale dell'impresa nell'era della globalizzazione

3 Il codice di condotta: nuove prospettive di autoregolamentazione

4 Responsabilità sociale dell'impresa e diritto del lavoro nel contesto italiano e flessibilità sociale dell'impresa nei Paesi dell'Unione Europea

Bibliografia 


\section{Organizzazione Internazionale del Lavoro e Nazioni Unite contro la discriminazione nel lavoro}

A partire dal 1919, anno della sua istituzione, l'Organizzazione Internazionale del Lavoro (OIT), che da sempre si occupa del riconoscimento universale e del rispetto dei diritti umani nel lavoro, ha assunto tra gli obiettivi cardine della sua attività (ribadendoli nella Dichiarazione di Philadelphia del 1944), quello di promuovere il rispetto dell'eguaglianza di opportunità e di trattamento nel lavoro e il perseguimento di condizioni eguali nell'accesso e nello svolgimento del rapporto di lavoro, a prescindere da razza, sesso e religione ${ }^{1}$.

Sulla base dei principi ${ }^{2}$ sanciti nello Statuto delle Nazioni Unite del 1945, nella Dichiarazione Universale dei Diritti dell'Uomo adottata nel 1948 dall'Assemblea delle Nazioni Unite e a seguire, nel 1966, nel Patto sui diritti civili e politici e nel Patto sui diritti economici, sociali e culturali ${ }^{3}$ adottati dalla stessa Assemblea Generale, si afferma e si sviluppa il principio di eguaglianza degli individui che si estende anche alla sfera lavorativa.

Gli strumenti internazionali adottati dalle Nazioni Unite e in particolare dall'OIT per fronteggiare la discriminazione nel campo del lavoro sono numerosi: la Convenzione OIT n. 100 del $1951^{4}$ sull'uguaglianza di retribuzione costituisce il primo esempio di strumento adottato a questo scopo, dopo il quale, però, si chiarisce ben presto la necessità di dover vietare preliminarmente altre forme di disparità al fine di poter realizzare nel concreto un'effettiva uguaglianza nella retribuzione. È stata così successivamente adottata e ampiamente ratificata la Convenzione OIT n. 111 del 1958 (e relativa Raccomandazione) concernente tutti i tipi di discriminazione nell'impiego e nell'occupazione ${ }^{5}$, che trova applicazione nei confronti dei lavoratori sia del settore pubblico che del settore privato, anche se non cittadini dello Stato membro in cui lavorano e nei confronti non solo degli individui già inseriti nel mondo del lavoro ma anche di coloro che intendono entrarvi, includendo l'accesso alla formazione

1 ACRI, D., La lotta internazionale contro la discriminazione nel lavoro in I diritti dell'uomo. Cronache e battaglie, 2/2004, p. 80.

2 Sono elencate diverse norme volte alla tutela contro la discriminazione nel lavoro che impongono agli Stati Membri di promuovere i diritti umani e le libertà fondamentali senza operare distinzioni di sesso, razza, lingua o religione (si vedano gli artt. 1; 13 par. 1 e 62 par. 2 e l'art. 68 in cui è attribuita al Consiglio Economico e Sociale la facoltà di creare commissioni per promuovere i diritti umani), cfr. ACRI, D., La lotta internazionale contro la discriminazione nel lavoro in I diritti dell'uomo. Cronache e battaglie, 2/2004, p. 83.

3 Insieme ai quali la Dichiarazione Universale dei Diritti dell'Uomo costituisce la c.d. Carta Internazionale dei Diritti dell'Uomo.

4 Con allegata Raccomandazione n. 90.

5 Convenzione che è stata alla base dell'azione OIL contro i regimi di apartheid del Sudafrica e della Namibia, cfr. ACRI, D., La lotta internazionale contro la discriminazione nel lavoro in I diritti dell'uomo. Cronache e battaglie, 2/2004, p. 83. 
professionale $^{6}$. In tale Convenzione in particolare si precisa il significato da attribuire al concetto di discriminazione, individuando all'art. 1 , par. $1 \mathrm{a}^{7}$, una serie di categorie alle quali è riconducibile, definendola come "ogni distinzione, esclusione o preferenza basata sulla razza, il colore, il sesso, la religione, l'opinione politica, la discendenza nazionale o l'origine sociale, che ha per effetto di negare o alterare l'uguaglianza di possibilità o di trattamento in materia di impiego o di professione".

The ILO outlines at least three different types of discrimination addressed by Convention 111. It describes direct discrimination as "rules, practices and policies which exclude or give preference to certain individuals just because they belong to a particular group" (International Labour Organization 2007:9). In contrast, it says, indirect discrimination occurs when neutral norms have a disproportionate impact on an identifiable group, without justification. The ILO specifically identifies the differential treatment of particular categories of workers, such as domestic workers, as a form of indirect discrimination that often affects "low-income women belonging to racial or ethnic minorities, and who are often foreigners" (International Labour Organization 2007:9). Finally, it describes structural discrimination as inherent or institutionalized social, institutional, and legal constructs that reflect and reproduce discriminatory practices and outcomes. In looking at how to eliminate discrimination, the ILO recognizes that certain differences in treatment are not discriminatory but are, in fact, necessary to achieve equality. "Special measures that entail nonidentical treatment of individuals with particular needs, owing to reasons such as their sex, mental, sensory or physical impairment or social origin, do not constitute discrimination. Giving effect to the principle of equal treatment and opportunities means more than treating persons in the same way; it also requires special measures and the accommodation of differences" (International Labour Organization 2007:10). The ILO also recognizes the importance of special temporary measures or affirmative action that might be necessary to "accelerate the pace of improvement of the situation of groups that are at a serious disadvantage because of past or present discrimination" (International Labour Organization 2007:10) ${ }^{8}$.

Con riferimento alla discriminazione nel lavoro per motivi razziali, si ricordano la Convenzione OIT n. 29 del 1930 sul lavoro forzato e obbligatorio e la Convenzione

6 Cfr. ACRI, D., La lotta internazionale contro la discriminazione nel lavoro in I diritti dell'uomo. Cronache e battaglie, 2/2004, p. 81.

7 Cfr. ACRI, D., La lotta internazionale contro la discriminazione nel lavoro in I diritti dell'uomo. Cronache e battaglie, 2/2004, p. 81.

8 ONTIVEROS, M. L., Employment Discrimination in GROSS, J. A. and COMPA, L. (edited by), Human Rights in Labor and Employment Relations : International and Domestic Perspectives, United States of America: Labor and Employment Relations Association, 2009, p. $199,200$. 
OIT n. 105 del 1957 sull'abolizione del lavoro forzato?.

Si rammenta infine la Convenzione n. 156 approvata dall'OIT nel 1981 al fine di promuovere la conciliazione tra gli impegni professionali e quelli familiari a favore dei lavoratori, anche nell'ottica di garantire l'eguaglianza tra i sessi, arrivando a parlare del Rapporto Generale sullo stato della discriminazione nel mondo del lavoro adottato dal Consiglio di Amministrazione dell'OIT in occasione della sua 289.ma sessione. Questo documento è collegato a "Time for equality at work" consapevolezza dell'intollerabilità della dilagante disparità, nelle sue numerose e varie forme, nell'impiego e nella professione, ponendo l'accento sulla peculiarità del lavoro che è quella di essere "punto d'ingresso privilegiato" per poter eliminare la discriminazione nella società ${ }^{11}$.

\section{La Responsabilità sociale dell'impresa nell'era della globalizzazione}

La c.d. "dimensione sociale della globalizzazione" costituisce oggi un tema sul quale si concentrano diverse discussioni che traggono origine dal generale malcontento derivante, da una parte, dall'incapacità della politica di condizionalità del Fondo monetario internazionale di conseguire risultati soddisfacenti sia per quanto riguarda la lotta alla povertà sia per quanto concerne la crescita economica e, dall'altra, dalla constatata irraggiungibilità di vantaggi concreti per i Paesi in via di sviluppo che hanno aderito all'Organizzazione mondiale del commercio (OMC $)^{12}$.

9 Tra gli strumenti adottati dalle Nazioni Unite contro la discriminazione razziale si ricordano: la Dichiarazione sull'eliminazione di tutte le forme di discriminazione razziale del 1963, la Convenzione sulleliminazione di tutte le forme di discriminazione razziale del 1965, la Convenzione per l'eliminazione e la punizione del crimine di apartheid del 1973, la Convenzione sull'eliminazione delle discriminazioni contro le donne del 1979, la Dichiarazione sull'eliminazione della violenza contro le donne del 1993, la Dichiarazione di Vienna adottata a conclusione della Conferenza Mondiale delle Nazioni Unite sui Diritti Umani (cfr. ACRI, D., La lotta internazionale contro la discriminazione nel lavoro in I diritti dell'uomo. Cronache e battaglie, 2/2004, p. 84).

10 Rapporto Globale sull'eliminazione della discriminazione in materia di impiego e professione presentato nel corso della 91.ma sessione (Ginevra, giugno 2003) della Conferenza Generale dal Direttore Generale dell'OIL che completa il primo ciclo quadriennale di Rapporti sulle categorie di diritti fondamentali sanciti dalla "Dichiarazione OIL sui principi e diritti fondamentali nel lavoro e suoi seguiti” del 1998, cfr. ACRI, D., La lotta internazionale contro la discriminazione nel lavoro in I diritti dell'uomo. Cronache e battaglie, 2/2004, p. 80.

11 ACRI, D., La lotta internazionale contro la discriminazione nel lavoro in I diritti dell'uomo. Cronache e battaglie, 2/2004, p. 80.

12 ADINOLFI, G., recensione a Claudio Di Turi Globalizzazione dell'economia e diritti umani fondamentali in materia di lavoro: il ruolo dell'OIL e dell'OMC in Diritti umani e diritto internazionale, 2 (2008), p. 465. 
Si sta facendo così strada l'idea che sia necessario dare maggiore rilevanza ad interessi di tipo sociale per garantire una distribuzione del benessere più egualitaria, anche grazie all'azione di sistemi normativi internazionali che consentano di appoggiare strategie di crescita economica adeguate a tale scopo.

Nell'attuale scenario, nell'ambito della cooperazione internazionale in materia commerciale, da un lato si collocano le attività promosse dall'OIT che, come si è potuto facilmente desumere dalla precedente panoramica normativa, hanno l'obiettivo di garantire una giustizia sociale, e, dall'altro, si hanno invece le attività dell'OMC finalizzate ad accrescere gli scambi di beni e servizi.

Oggetto di particolare attenzione risulta oggi il rapporto intercorrente tra i due tipi di finalità perseguite da OIT e OMC nei loro rispettivi operati e nella dottrina internazionalistica. In special modo, è in corso una discussione sulla legittimità di possibili restrizioni degli scambi internazionali ad opera di stati membri dell'OMC verso certi Paesi in cui si è verificato il mancato rispetto di quanto disposto dalle convenzioni OIT in tema di diritti fondamentali dei lavoratori ${ }^{13}$.

In questo contesto, continuando a restare sul piano globale, una prospettiva interessante può essere rappresentata dal ruolo della responsabilità sociale dell'impresa (Rsi o Csr secondo l'acronimo inglese), la quale, dal punto di vista giuridico, interessa la sfera dei diritti dell'uomo e, dal punto di vista economico, la sfera della liberalizzazione del commercio mondiale ${ }^{14}$.

L'applicazione di prassi di Rs a livello internazionale, verso cui le imprese di tutto il mondo si stanno avviando, costituisce infatti una possibile via di uscita dai problemi sorti dalla condotta collaudata dei Paesi industrializzati che da sempre pretendono dai Paesi in via di sviluppo che essi si conformino alle proprie condizioni sociali della produzione $\mathrm{o}$ ad ogni modo a condizioni più elevate, perlomeno pari ai livelli degli standard internazionali elaborati in sede Oit e Ocse ${ }^{15}$.

Per ovvie ragioni, la prima e maggiore sfida a cui va incontro la Rs è quella nel Sud e nell'Est del mondo, innanzitutto per una questione di tipo etico. Infatti, c'è da domandarsi se davvero le multinazionali, in quest'era della globalizzazione e della liberalizzazione, saranno in grado di fare della Rs uno strumento vantaggioso anche per i Paesi in via di sviluppo e non solo per i Paesi industrializzati in cui vige una concezione di consumo critico ormai piuttosto consolidata.

13 ADINOLFI, G., recensione a Claudio Di Turi Globalizzazione dell'economia e diritti umani fondamentali in materia di lavoro: il ruolo dell'OIL e dell'OMC in Diritti umani e diritto internazionale, 2 (2008), p. 465.

14 TURSI, A., Responsabilità sociale dell'impresa, «etica d'impresa» e diritto del lavoro, in Lavoro e Diritto, a. XX, n.1, inverno 2006, p. 78.

15 TURSI, A., Responsabilità sociale dell'impresa, «etica d'impresa» e diritto del lavoro, in Lavoro e Diritto, a. XX, n.1, inverno 2006, p. 78. 
Questo interrogativo, poi, è maggiormente supportato dal fatto che nel Sud del mondo i principi di liberalizzazione e integrazione dei mercati non sono gli stessi e non esiste nemmeno un confronto immediato con quei consumatori che hanno la possibilità di pagare di più per ottenere un prodotto proveniente da un'impresa socialmente responsabile ${ }^{16}$.

Il motivo che conduce progressivamente all'adozione di pratiche di Rs da parte delle imprese globali, si sostanzia nel fatto che sono sempre di più i consumatori che, nei Paesi industrializzati, sviluppano una sensibilità etica al consumo e che, in questo senso, pretendono un certo tipo di risposta in termini di produzione, la quale, a sua volta, deve conformarsi a livello globale alla qualità della domanda.

Attraverso una sistematizzazione organica del lavoro a livello mondiale si consente di coniugare efficienza e quantità nel lavoro e, in questo modo, il livello di produttività nei Paesi in via di sviluppo risulta assai alto, complici ovviamente il basso costo del lavoro e le condizioni in cui viene esercitato.

È un modello che concilia alti standard qualitativi con un basso costo del lavoro, che le imprese globali americane ed europee hanno in un certo senso imposto, attraverso politiche di Rsi che possono spesso definirsi «da manuale», oltre che pionieristiche, e che si sta diffondendo e consolidando per via emulativa. Si potrebbe anzi affermare che, oggi, le imprese più «socialmente responsabili» sono proprio le più «globalizzate» ${ }^{17}$.

Occorre però mettere in dubbio, come in parte si sostiene oggi, che diritto ed etica camminino "sulle gambe dell'economia"18 mondiale: prassi di Rs applicate ai Paesi in via di sviluppo, ad esempio, dovrebbero essere giustificate da motivazioni etico- sociali più che di tipo economico perché se si concepisse una Rs fondata unicamente sulle conseguenze economiche pretese dall'attività d'impresa, essa risulterebbe completamente estranea alle concrete necessità delle persone.

16 Rebaudengo, P. A., Prefazione a Montuschi L. e Tullini P. (a cura di) Lavoro e responsabilità sociale dell'impresa, Bologna: Zanichelli, 2006.

17 TURSI, A., Responsabilità sociale dell'impresa, «etica d'impresa» e diritto del lavoro, in Lavoro e Diritto, a. XX, n.1, inverno 2006, p. 79.

18 TURSI, A., Responsabilità sociale dell'impresa, «etica d'impresa» e diritto del lavoro, in Lavoro e Diritto, a. XX, n.1, inverno 2006, p. 79. 


\section{Il codice di condotta: nuove prospettive di autoregolamentazione}

Continuando a parlare di globalizzazione economica, essa, in tema di regolazione, ha posto una serie di questioni rimaste in parte ancora aperte e, in questo contesto, un ruolo importante può essere giocato da uno strumento quale il codice di condotta, che si colloca ambiguamente tra la sfera morale e quella del diritto positivo.

Il codice è "esemplare di questa tensione tra diritto e non diritto condensata nel tema della responsabilità sociale dell'impresa" ${ }^{\prime 19} \mathrm{e}$, se da un lato può essere oggetto di processi di giuridificazione ovvero di contrattualizzazione, dall'altro può essere impiegato come utile supporto a norme internazionali, soprattutto quelle OIT, andando ad espletare funzioni normative di appoggio a sistemi che in particolar modo nei Paesi in via di sviluppo risultano lacunosi nella tutela dei diritti sociali, consentendo di andare oltre i limiti di territorialità delle leggi.

Gli ideatori del codice etico dell'impresa devono creare un "credo" che venga facilmente interpretato, chiaro nelle sue premesse e con lo scopo di perseguire uno spirito di cooperazione tra tutti i partecipanti dell'impresa ${ }^{20}$.

La Rsi, delle cui prassi i codici di condotta rappresentano lo strumento per eccellenza, fa parte di un processo di "integrazione sociale dell'economia"21 così pregnante da essere per forza di cose legato indissolubilmente alla disciplina del diritto del lavoro ${ }^{22}$ delineando così una profonda integrazione con la prospettiva giuslavoristica, andando a regolare il comportamento dell'impresa nell'ottica di interessi e valori che si allargano anche alla sfera delle istanze di natura sociale.

L'utilizzo dei codici di condotta si presta a diverse chiavi di lettura che portano a domandarsi se la loro adozione, simbolo di una regolamentazione dotata dei caratteri della volontarietà, flessibilità e non vincolatività, possa condurre all'assunzione della medesima efficacia della norma legale imposta da uno Stato al proprio territorio nazionale, tenendo ben presente che "il codice non deve essere una vaga proposizione, bensì un documento ufficiale nel quale vengono rispettate le aspettative pubbliche relative al comportamento morale dell'impresa"23.

19 PERUlli, A., in Prefazione a Perulli A. (a cura di), L'impresa responsabile. Diritti sociali e corporate social responsability, Matelica: Halley Editrice, 2007.

20 BARDA, G. e OSTAPSKI, S. A., La Revisione Etica dell'Impresa in Etica degli affari e delle professioni, 2/93, p. 49.

21 PERULLI, A., Corporate Social Responsibility e diritto del lavoro, in PERULLI, A. (a cura di) L'impresa responsabile. Diritti sociali e corporate social responsability, Matelica: Halley Editrice, 2007, p. 98.

22 Sul rapporto tra Rsi e diritto del lavoro nel contesto italiano si tornerà nell'ultimo capitolo di questo articolo.

23 BARDA, G. e OSTAPSKI, S. A., La Revisione Etica dell'Impresa in Etica degli affari e delle 
Occorre sottolineare che i codici di condotta rappresentano strumenti volontari di regolazione che una volta adottati ammettono numerose possibilità di applicazione, che non escludono la transazione da una dimensione prettamente privatistica verso una dimensione di regolazione pubblica del comportamento d'impresa, testimoniando un'apertura cognitiva del sistema giuridico comunque ancorato alla sua ben definita sfera normativa ${ }^{24}$.

I codici di condotta, in questo modo, possono rivestire un ruolo non marginale nel cammino volto alla ricerca di una "governance mondiale" in formazione, "multipolare e a-sistemica, che sancisce nell'era della globalizzazione il senso della connessione tra economia e valori sociali" ${ }^{25}$.

La Rsi necessita di una sistematizzazione di quelle che possiamo definire come sue fonti normative, soprattutto alla luce del fatto che sono diversi i soggetti, dalle società multinazionali alle Ong, che ricorrono a una copiosa adozione di codici di condotta, i quali, debbono essere sottoposti a una distinzione da effettuare in ragione della loro fonte, dell'oggetto che li caratterizza e degli scopi perseguiti.

La prima distinzione riguarda i codici interni o individuali e i codici esterni.

I primi concorrono alla gestione e organizzazione dell'azienda esprimendo quelle che sono le politiche aziendali e le norme di comportamento alle quali i dipendenti debbono conformarsi e, in generale, rappresentano una categoria assai varia sia per quanto riguarda la molteplicità dei testi che la loro compiutezza.

Il processo seguito è molto libero e varia a seconda delle tipologie di imprese e dei settori produttivi considerati. La proprietà o la dirigenza utilizzano i codici interni, che costituiscono documenti ufficiali da loro elaborati ed adottati, come strumento per riunire in maniera organica e diffondere i principi su cui si basa la cultura d'impresa, sia al personale interno che ai principali interlocutori esterni2 ${ }^{26}$.

I secondi, i codici esterni, che si distinguono, a seconda della fonte, in diverse tipologie $e^{27}$, sono così definiti perché la loro elaborazione dipende da soggetti non

professioni, 2/93, p. 49.

24 PERULLI, A., Corporate Social Responsibility e diritto del lavoro, in PERULLI, A. (a cura di), L'impresa responsabile. Diritti sociali e corporate social responsability, Matelica: Halley Editrice, 2007, p. 120.

25 PERULLI, A., Corporate Social Responsibility e diritto del lavoro in PERULLI, A. (a cura di), L'impresa responsabile. Diritti sociali e corporate social responsability, Matelica: Halley Editrice, 2007, p. 120.

26 PERULLI, A., Corporate Social Responsability e diritto del lavoro, in PERULLI, A. (a cura di), L'impresa responsabile. Diritti sociali e corporate social responsability, Matelica: Halley Editrice, 2007, p. 111,112.

27 Codici formulati da imprese internazionali; codici di condotta di origine statale ed interstatale; codici di condotta di origine privatistica. 
rientranti nel campo di governo dell'impresa (presentando così la prerogativa, a differenza di quelli interni, di corrispondere a modelli omogenei a portata generale che si rifanno a norme di diritto internazionale), e si caratterizzano per l'adesione volontaria dei loro destinatari.

Queste caratteristiche di generalità ed astrattezza del codice sono particolarmente importanti nella prospettiva della regolazione (dei comportamenti dell'impresa) nella catena di produzione su scala globale. In questa prospettiva, l'enunciazione di principi generali, così come i richiami alla legislazione interna o internazionale, possono avere un rilievo inaspettato: lungi dall'essere pleonastici, quei richiami rivelano una realtà in cui le norme di diritto statale o interstatale non vengono spontaneamente rispettate (si pensi alle convenzioni OIT non ratificate o, se ratificate, non applicate), con tutte le implicazioni tipiche della crisi di effettività della norma giuridica nel nostro tempo. Quel richiamo può perseguire, allora, quantomeno un obiettivo pedagogico, contribuendo a rendere effettiva l'interiorizzazione, da parte dei destinatari del codice collettivo, dei valori tutelati dal diritto statale o internazionale ${ }^{28}$.

Un particolare esempio di trasparenza ed effettività nei confronti delle norme stabilite nel codice stesso è dato da un tipo di codice esterno, il codice di condotta su base negoziata, che deve tali meriti al fatto di essere elaborato in sede di contrattazione collettiva, a livello nazionale o sopranazionale, coinvolgendo in questo modo direttamente e bilateralmente gli attori collettivi negli accordi e nella formulazione delle norme di condotta che le imprese multinazionali potranno poi adottare ${ }^{29}$.

Con la locuzione "normazione tecnica" si suole definire la produzione o l'omologazione di norme tecniche alle quali sono chiamati a rispondere prodotti, servizi o procedure di fabbricazione. Sulla base di essa si formulano i codici tecnici, imprescindibili dall'attività di certificazione sociale volta alla valutazione positiva di conformità alle norme tecniche, iquali ricontestualizzano in ambito giuridico, economico e politico gli standard tecnici di produzione elaborati a livello transnazionale ${ }^{30}$.

La sigla SA 8000 (Social Accountability 8000) racchiude gli standard provenienti dalle norme tecniche che si riferiscono alla materia sociale. Esse si richiamano direttamente alle convenzioni OIT, hanno portata globale e sono complementari

28 PERULLI, A., Corporate Social Responsability e diritto del lavoro, in PERULLI, A. (a cura di), L'impresa responsabile. Diritti sociali e corporate social responsability, Matelica: Halley Editrice, 2007, p. 113.

29 PERULLI, A., Corporate Social Responsability e diritto del lavoro, in PERULLI, A. (a cura di), L'impresa responsabile. Diritti sociali e corporate social responsability, Matelica: Halley Editrice, 2007, p. 113.

30 PERULLI, A., Corporate Social Responsibility e diritto del lavoro in PERULLI, A. (a cura di), L'impresa responsabile. Diritti sociali e corporate social responsability, Matelica: Halley Editrice, 2007, p. 114. 
alla legge nazionale la quale, secondo quanto autorizzato alle imprese da SA 8000 nel richiamare il principio di favore, va applicata solamente quando risulta essere più favorevole rispetto agli standard tecnici.

Occorre infine sottolineare il fatto che le norme tecniche, diversamente dai codici di condotta, "non possono dunque generare regressioni dei diritti individuali dei lavoratori; al contrario, intendono contribuire alleffettività delle norme sociali fondamentali completando la legge nazionale alla quale le imprese accreditate sono sottomesse" ${ }^{\prime 31}$.

Un problema non piccolo deriva dal fatto che esaminare l'attuazione dei codici di condotta non risulta particolarmente agevole.

La prima caratteristica del codice di condotta che impone la necessità di un'impellente intervento di mezzi giuridici che consentano l'attuazione delle obbligazioni sancite dall'impresa nel codice medesimo, si riferisce al fenomeno dell'autoregolazione che fa in modo che le norme del codice di condotta non abbiano come destinatario un soggetto di diritto nel senso stretto del termine.

Infatti, nella sfera dell'autoregolazione, il confine che intercorre tra soggetto di diritto e autore della regola è assai labile, tanto che i due finiscono per confondersi e a partire da ciò si configura un iniziale limite di effettività, alquanto determinante.

Sono tre le condizioni che consentono al codice di acquisire l'effettività necessaria $^{32}$ : la prima risiede nella pubblicità delle norme nei confronti di tutti i possibili stakeholder, dai lavoratori ai consumatori, passando per i fornitori, i subfornitori, i partners commerciali, i governi stranieri, garantendo così trasparenza e conoscibilità al codice di condotta.

Il controllo, che può essere di tre tipi, rappresenta la seconda condizione perché il codice possa raggiungere un livello di effettività sufficiente. Si parla di controllo endogeno (il più frequente) quando è l'impresa ad effettuarlo dando luogo ad un'attività di auto-certificazione; di controllo esogeno quando esso viene esercitato da soggetti esterni all'impresa; infine, si può anche avere un controllo di tipo misto.

La terza condizione per poter parlare di effettività è data dalla presenza di un apparato sanzionatorio o, quantomeno, di rimedi giuridici, in grado di garantire il rispetto delle norme del codice che, come è risaputo, rappresentano obblighi di tipo volontario derivanti dalla stesse imprese che le adottano.

31 PERULLI, A., Corporate Social Responsibility e diritto del lavoro in PERULLI, A. (a cura di), L'impresa responsabile. Diritti sociali e corporate social responsability, Matelica: Halley Editrice, 2007, p. 114.

32 PERULLI, A., Corporate Social Responsibility e diritto del lavoro in PERULLI, A. (a cura di), L'impresa responsabile. Diritti sociali e corporate social responsability, Matelica: Halley Editrice, 2007, p. 116. 
A questo proposito, occorre operare una distinzione tra quella che è una sanzione giuridica, la quale attiva rimedi sanzionatori in caso di mancato rispetto di un diritto, da quella che è una sanzione sociale, descrivibile come l'espressione dei modi di reagire di una data società al fine di garantire un controllo capillare sui suoi membri.

Nello stesso diritto internazionale economico è possibile constatare una certa cedevolezza delle sanzioni giuridiche a favore di sanzioni informali, rapportabili alla sanzione sociale: evidentemente, l'autoregolazione partecipa a una dimensione di "informalismo sanzionatorio", in quanto combina sanzione sociale e sanzione economica ${ }^{33}$.

Restando sempre in tema di autoregolamentazione, un'osservazione a parte merita la convinzione insita nella nostra cultura che il diritto preceda l'etica ovvero che sia sufficiente ricorrere all'applicazione di norme per prevenire l'accadimento di qualsiasi fenomeno.

A fronte di questa convinzione, è invece possibile dimostrare il contrario, cioè che è il diritto che segue l'etica e questo accade per due motivi: il primo consiste nel fatto che è l'etica che consente di stabilire se le leggi sono giuste o meno e il secondo risiede nell'importanza dell'autoregolamentazione che a differenza del diritto, risulta essere lo strumento più indicato per evitare il verificarsi di una serie di comportamenti sbagliati ${ }^{34}$.

Più precisamente, a questo proposito si può fornire una duplice argomentazione della tesi in base a cui è l'etica a precedere il diritto: occorre in primis considerare due prospettive, quella del cittadino e quella del legislatore, per affermare che l'etica, nella sua accezione pubblica, precede la legge. Essa, per il cittadino che sa di essere sottomesso alle norme giuridiche, consente, stabilendo quel parametro che permette di discernere ciò che è giusto da ciò che non lo è, di legittimare ai suoi occhi la pretesa di autorità della legge alla quale egli si deve conformare, mentre per il legislatore l'etica definisce le linee guida per la creazione dei meccanismi istituzionali che consentono l'elaborazione delle decisioni pubbliche che hanno l'obiettivo di essere condivise da un'intera società ${ }^{35}$.

Secondariamente, se si analizza l'etica nella sua accezione di etica degli affari che riguarda l'emanazione di codici di autoregolazione, si può affermare che l'autoregolamentazione, come sopra anticipato, consente di spingersi fin dove il diritto non è in grado di arrivare, "ovvero a eliminare forme di opportunismo che

33 PERULLI, A., Corporate Social Responsibility e diritto del lavoro in PERULLI, A. (a cura di),

L'impresa responsabile. Diritti sociali e corporate social responsability, Matelica: Halley Editrice, 2007, p. 118.

34 SACCONI, L., Etica e Legge: quale rapporto? in Etica degli affari e delle professioni, 2/93, p. 4.

35 SACCONI, L., Etica e Legge: quale rapporto? in Etica degli affari e delle professioni, 2/93, p. 5. 
persistono anche sotto istituzioni economiche progettate in modo da provvedere incentivi, e che continuano perciò a generare iniquità di trattamento e perdite di efficienza congiunta, e alla fin fine destabilizzano quelle stesse istituzioni" ${ }^{36}$.

L'adozione di un codice di autoregolazione sembra costituire la risposta più giusta per consentire a tutti i possibili stakeholder di un'impresa di cooperare con coloro che esercitano potere discrezionale, soggetti a loro volta agli obblighi di responsabilità sanciti nel codice, i quali limitano e circoscrivono l'uso della discrezionalita ${ }^{37}$.

Lo strumento che contribuisce all'individuazione dei doveri di responsabilità sanciti poi all'interno dei codici etici che regolamenteranno l'attività di imprese e le professioni, è il contratto sociale e la peculiarità dei codici di autoregolazione risiede nel fatto "di dare incentivi ex post all'osservanza di tali doveri”"38, ovvero di non fermarsi alla semplice enunciazione di essi.

L'obiettivo primario che porta all'adozione di un codice etico, per ricollegarsi a quanto esposto nella premessa di questo ragionamento, è quello di garantire la cooperazione e in quest'ottica, la salvaguardia della reputazione che deriva dal rispetto di quanto stabilito dal codice, è la condicio sine qua non per riuscire a continuare a cooperare, nella convinzione che "l'impresa, con il rispetto della legge e del comportamento etico, può ottenere il sostegno della società e, tramite questo, raggiungere il successo economico e la libertà d'azione" ${ }^{\prime 39}$.

\section{Responsabilità sociale dell'impresa e diritto del lavoro nel contesto italiano e flessibilità sociale dell'impresa nei Paesi dell’Unione Europea}

Come in precedenza anticipato, per concludere, si farà una breve riflessione concernente i rapporti tra Rsi e diritto del lavoro nel contesto italiano e, infine, ci sarà un richiamo generale ${ }^{40}$ alla flessibilità del mercato del lavoro, considerando un particolare profilo riguardante la responsabilità dell'impresa connessa alla capacità di rispondere alle domande di flessibilità sociale ${ }^{41}$ dei Paesi dell'Unione Europea.

SACCONI, L., Etica e Legge: quale rapporto? in Etica degli affari e delle professioni, 2/93, p. 5.

SACCONI, L., Etica e Legge: quale rapporto? in Etica degli affari e delle professioni, 2/93, p. 11.

38 SACCONI, L., Etica e Legge: quale rapporto? in Etica degli affari e delle professioni, 2/93, p. 13.

39 BARDA, G. e OSTAPSKI, S. A., Le Revisione Etica dell'Impresa in Etica degli affari e delle professioni, 2/93, p. 47.

40 Ed esterno seppur connesso al concetto di Rsi.

${ }^{41}$ SEMENZA, R., Le trasformazioni del lavoro. Flessibilità, disuguaglianze, responsabilità dell'impresa, Roma: Carrocci, 2004, p. 158. 
Per quanto riguarda il primo punto, si può affermare che la Rsi rappresenti per il diritto del lavoro italiano sia un'opportunità che una sfida teorica non irrilevante ${ }^{42}$.

La nota definizione di $\mathrm{Csr}^{43}$ derivante dal Libro Verde del 2001 costituisce per il giurista una sfida non piccola in termini metodologici. Per prima cosa, infatti, la Csr, nella sua definizione classica, configura un tipo di regolazione (se si vuole adottare questo termine) economica o sociologica ma non giuridica, richiedendo in questo modo l'internalizzazione della norma ${ }^{44}$.

In Italia, la cultura lavoristica si fonda sulla concezione in base alla quale la regolazione normativa costituisce l'unico strumento in grado di condurre all'attuazione di determinati comportamenti e nonostante ci sia stata un'evoluzione a riguardo, questa impostazione non è stata lo stesso superata.

Il tentativo della Csr è, per il momento, quello di apportare un contributo alla regolazione classica ma senza sostituirla, anche se implicitamente porta con sé il messaggio che i diritti, un domani, potrebbero non essere più necessari, se gli imprenditori si renderanno conto che converrà loro porre in essere condotte cooperative e non opportunistiche ${ }^{45}$ per realizzare i propri obiettivi produttivi. Infatti, la Csr mira ad alimentare nella classe imprenditoriale una nuova concezione della produttività e della competitività improntate su principi di cooperazione $\mathrm{e}$ responsabilità sociale, andando in questo modo immancabilmente ad incidere sul diritto del lavoro, ben sapendo che ciò comporterà a posteriori la necessità di un assestamento sia di tipo normativo che, soprattutto, di tipo culturale.

L'altra sfida che la Rsi costituisce per il diritto del lavoro è rappresentata dal fatto che il tipo di cooperazione contemplata dalle prassi di Rsi è onnicomprensiva, investe ogni campo. Fino ad ora in Italia i lavoratori subordinati hanno rappresentato lo stakeholder principale, così come del resto è previsto dalla stessa Costituzione della Repubblica italiana (1948), che mette al primo posto i diritti dei lavoratori rispetto a tutti gli altri soggetti ugualmente interni, seppur a titoli diversi, all'attività d'impresa. Non è previsto un criterio di base da parte della stakeholder theory rivolto alla composizione dei conflitti di interesse interni al gruppo degli stakeholder non controllanti ${ }^{46}$. "Tuttavia, anche ammesso che gli stakeholder interni possano essere

42 DEL PUNTA, R., Responsabilità sociale dell'impresa e diritto del lavoro in Lavoro e Diritto, a. XX n. 1, inverno 2006, p. 58.

43 «L'integrazione su base volontaria, da parte delle imprese, delle preoccupazioni sociali ed ecologiche nelle loro operazioni commerciali e nei rapporti con le parti interessate».

44 DEL PUNTA, R., Csr, organizzazione e qualità del lavoro in MONTUSCHI, L. e TULLINI, P. (a cura di), Lavoro e responsabilità sociale dell'impresa, Bologna: Zanichelli, 2006, p. 4.

45 DEL PUNTA, R., Csr, organizzazione e qualità del lavoro in MONTUSCHI, L. e TULLINI, P. (a cura di), Lavoro e responsabilità sociale dell'impresa, Bologna: Zanichelli, 2006, p. 6.

46 DEL PUNTA, R., Csr, organizzazione e qualità del lavoro in MONTUSCHI, L. e TULLINI, 
preferiti a quelli esterni”, "già il fatto di porre i due gruppi sullo stesso piano concettuale, e dunque in potenziale concorrenza, costituisce, per il giuslavorista, un'eresia o poco di meno" 47 .

Trattando non solamente dell'Italia e andando al di là dell'ambito connesso alla Rsi ma parlando sempre di lavoro, si conclude con un ragionamento connesso alla c.d. flessibilità sociale dell'impresa, intendendo con ciò la capacità (oltre che la predisposizione) di un'impresa di adattare la sua attività in modo da riuscire ad andare incontro alla crescente flessibilità necessaria a una società sottoposta a continua trasformazione, consentendo di armonizzare i delicati rapporti tra sfera lavorativa e non lavorativa degli individui ${ }^{48}$ nel contesto europeo.

Il livello di produttività è indubbiamente connesso alla forza lavoro e alle modalità con cui vengono combinate le competenze e le qualificazioni che portano così a performance sempre diverse tra loro.

Come dimostrano recenti studi riferiti al contesto europeo concernenti i problemi di labour shortages, skill shortages and gaps, è necessario da parte di tutti gli attori interessati ovvero imprese, terzo settore, famiglie e istituzioni realizzare un'integrazione delle politiche nell'ottica di una partnership allargata ${ }^{49}$.

Così, se si considerasse che flessibilità sociale e flessibilità di mercato costituiscono due dimensioni strategiche che si collocano su livelli sociali e temporali diversi, pur dialogando e tendendo l'una verso l'altra, dal momento che all'interno dell'Unione Europea si collocano da un lato paesi, come la Gran Bretagna, con una forte flessibilità di mercato e una bassa flessibilità sociale e dall'altro paesi come la Germania, con una bassa flessibilità di mercato e un'elevata flessibilità sociale, si potrebbe ipotizzare di raggruppare i paesi secondo il criterio di rispondenza delle loro imprese alle esigenze sociali degli individui, connesse al lavoro e all'occupazione, considerando sempre il contesto istituzionale e le politiche di welfare e del lavoro praticate ${ }^{50}$.

P. (a cura di), Lavoro e responsabilità sociale dell'impresa, Bologna: Zanichelli, 2006, p. 8.

47 DEL PUNTA, R., Csr, organizzazione e qualità del lavoro in MONTUSCHI, L. e TULLINI, P. (a cura di), Lavoro e responsabilità sociale dell'impresa, Bologna: Zanichelli, 2006, p. 8.

48 SEMENZA, R., Le trasformazioni del lavoro. Flessibilità, disuguaglianze, responsabilità dell'impresa, Roma: Carrocci, 2004, pagg. 170,171.

49 SEMENZA, R., Le trasformazioni del lavoro. Flessibilità, disuguaglianze, responsabilità dell'impresa, Roma: Carrocci, 2004, pag. 172.

50 SEMENZA, R., Le trasformazioni del lavoro. Flessibilità, disuguaglianze, responsabilità dell'impresa, Roma: Carrocci, 2004, pag. 172. 


\section{Bibliografia}

ACRI, D., La lotta internazionale contro la discriminazione nel lavoro, in I diritti dell'uomo. Cronache e battaglie, n. 2/2004, p. 80 .

ADINOLFI, G., recensione a Claudio Di Turi, Globalizzazione dell'economia e diritti umani fondamentali in materia di lavoro: il ruolo dell'OIL e dell'OMC, in Diritti umani e diritto internazionale, 2 (2008), p. 465.

BARDA, G. e OSTAPSKI, S. A., La Revisione Etica dell'Impresa, in Etica degli affari e delle professioni, 2/93, p. 47.

DEL PUNTA, R., Responsabilità sociale dell'impresa e diritto del lavoro, in Lavoro e Diritto, 2006, p. 41.

GROSS, J. A. and COMPA, L. (edited by), Human Rights in Labor and Employment Relations: International and Domestic Perspectives, United States of America, 2009.

MONTUSCHI, L. e TULLINI, P. (a cura di), Lavoro e responsabilità sociale dell'impresa, Bologna, 2006. PERULLI, A. (a cura di), L'impresa responsabile. Diritti sociali e corporate social responsability, Matelica, 2007.

SACCONI, L., Etica e Legge: quale rapporto?, in Etica degli affari e delle professioni, 2/93, p. 4.

SEMENZA, R., Le trasformazioni del lavoro. Flessibilità, disuguaglianze, responsabilità dell'impresa, Roma, 2004.

TURSI, A., Responsabilità sociale dell'impresa, «etica d'impresa» e diritto del lavoro, in Lavoro e Diritto, 2006, p. 65. 